\title{
Antibiotic resistance of pathogenic Acinetobacter species and emerging combination therapy
}

\author{
Bora Shin and Woojun Park \\ Laboratory of Molecular Environmental Microbiology, Department of \\ Environmental Science and Ecological Engineering, Korea University, \\ Seoul 02841, Republic of Korea \\ (Received July 17, 2017 / Revised Sep 19, 2017 / Accepted Sep 20, 2017)
}

The increasing antibiotic resistance of Acinetobacter species in both natural and hospital environments has become a serious problem worldwide in recent decades. Because of both intrinsic and acquired antimicrobial resistance (AMR) against last-resort antibiotics such as carbapenems, novel therapeutics are urgently required to treat Acinetobacter-associated infectious diseases. Among the many pathogenic Acinetobacter species, $A$. baumannii has been reported to be resistant to all classes of antibiotics and contains many AMR genes, such as $b l a_{\mathrm{ADC}}$ (Acinetobacter-derived cephalosporinase). The AMR of pathogenic Acinetobacter species is the result of several different mechanisms, including active efflux pumps, mutations in antibiotic targets, antibiotic modification, and low antibiotic membrane permeability. To overcome the limitations of existing drugs, combination theraphy that can increase the activity of antibiotics should be considered in the treatment of Acinetobacter infections. Understanding the molecular mechanisms behind Acinetobacter AMR resistance will provide vital information for drug development and therapeutic strategies using combination treatment. Here, we summarize the classic mechanisms of Acinetobacter AMR, along with newly-discovered genetic AMR factors and currently available antimicrobial adjuvants that can enhance drug efficacy in the treatment of $A$. baumannii infections.

Keywords: Acinetobacter, multidrug resistance, biofilm, membrane permeability, natural compounds, adjuvants

\section{Introduction}

The increasing concentration of antibiotics in the natural environment is of great concern because it can exert a selective pressure, which facilitates the horizontal gene transfer (HGT) of antimicrobial resistance (AMR) among microorganisms,

${ }^{\star}$ For correspondence. E-mail: wpark@korea.ac.kr; Tel.: +82-2-3290-3067;

Fax: +82-2-953-0737

Copyright (C) 2017, The Microbiological Society of Korea thus contributing to the spread of multidrug-resistant (MDR) bacteria. The number of infectious diseases caused by MDR bacteria has risen worldwide and become a major public health concern (Blair et al., 2015). Many pathogenic Acinetobacter species, particularly A. baumannii, have emerged as serious human health risks and are associated with many human diseases such as pneumonia, wound infections, meningitis, and sepsis. Their intrinsic genetic makeup and rapid evolution through induced mutagenesis and HGT may contribute to the ecological success of Acinetobacter species as opportunistic pathogens (Poirel et al., 2011; Stogios et al., 2017). The genus Acinetobacter currently comprises 51 genomic species, of which 20 are both categorized as pathogens and found in humans (Table 1). Acinetobacter species are a common causative agents of human infections in hospitals which include A. baumannii, A. pitti, and A. nosocomialis, the most frequently isolated species in hospital patients; as a result, these have been widely examined in recent years (Jung and Park, 2015) (Table 1). A. baumannii forms an A. baumannii complex with A. nosocomialis and A. pittii, which accounts for most (90-95\%) clinically significant infections (Nemec et al., 2011). Over the past 5 years, several novel Acinetobacter species of human origin have been described: A. seifertii, $A$. variabilis, $A$. proteolyticus, $A$. vivianii, and $A$. modestus (Table 1 ), which indicates possible discovery of more disease-causing Acinetobacter species in the future.

Acinetobacter baumannii possesses a range of intrinsic AMR genetic elements and shows high genome plasticity. In addition, recent data has demonstrated that this species has acquired and accumulated AMR determinants from other clinically important Gram-negative species such as Escherichia, Salmonella, and Pseudomonas species (Poirel et al., 2011). A. baumannii uses several intrinsic and acquired AMR mechanisms such as (i) increased production of antibiotic efflux pumps, (ii) adaptive evolution that generates point mutations in target proteins to avoid antibiotic action, (iii) enzymatic modification, and degradation of antibiotics, and (iv) reduction of membrane permeability. In many A. baumannii isolates, drug-resistant genes are clustered together into antibiotic resistance islands known as A. baumannii Resistant Islands (AbaRs) which accumulate in specific genetic regions such as comM (Pagano et al., 2016). Many variants of AbaRs have been documented, each differing in the number and nature of the genes involved in antibiotic resistance and transposons events. Many antibiotic resistance genes such as tetA(A), $a p h A 1 b$, aacC1, and aadA1 can be found in these AbaR regions (Post et al., 2010). These AbaR-type genome islands 
Table 1. Focus of research on clinically isolated Acinetobacter species

\begin{tabular}{|c|c|c|c|c|c|}
\hline \multirow{2}{*}{ Clinical isolate species } & \multicolumn{4}{|c|}{ Number of papers* } & \multirow{2}{*}{ Total $^{*}$} \\
\hline & $1948-1999$ & $2000-2005$ & $2006-2011$ & $2012-2017$ & \\
\hline Acinetobacter baumannii & 376 & 579 & 1,919 & 3,532 & 6,406 \\
\hline Acinetobacter calcoaceticus & 1,057 & 169 & 188 & 223 & 1,637 \\
\hline Acinetobacter lwoffii & 74 & 47 & 65 & 78 & 264 \\
\hline Acinetobacter baylyi & - & 4 & 97 & 87 & 188 \\
\hline Acinetobacter haemolyticus & 67 & 24 & 30 & 52 & 173 \\
\hline Acinetobacter johnsonii & 47 & 34 & 26 & 50 & 157 \\
\hline Acinetobacter junii & 35 & 26 & 37 & 46 & 144 \\
\hline Acinetobacter radioresistens & 25 & 30 & 22 & 33 & 110 \\
\hline Acinetobacter pittii & - & - & 6 & 99 & 105 \\
\hline Acinetobacter nosocomialis & - & - & 7 & 96 & 103 \\
\hline Acinetobacter ursingii & - & 2 & 10 & 12 & 24 \\
\hline Acinetobacter guillouiae & - & - & 2 & 18 & 20 \\
\hline Acinetobacter bereziniae & - & - & 2 & 17 & 19 \\
\hline Acinetobacter schindleri & - & 1 & 5 & 10 & 16 \\
\hline Acinetobacter parvus & - & 1 & - & 9 & 10 \\
\hline Acinetobacter seifertii & - & - & - & 10 & 10 \\
\hline Acinetobacter variabilis & - & - & - & 5 & 7 \\
\hline Acinetobacter proteolyticus & - & - & - & 1 & 1 \\
\hline Acinetobacter vivianii & - & - & - & 1 & 1 \\
\hline Acinetobacter modestus & - & - & - & 1 & 1 \\
\hline
\end{tabular}

* Number of papers in PubMed. Papers might be overlapped when different species are studied in the same paper.

have also been reported in other Acinetobacter strains including A. nosocomialis and A. seiffertii (Pagano et al., 2016). These AbaR regions are thought to have emerged from the integration of plasmids or other mobile elements (Post et al., 2010). This HGT may result in the generation of AMR hotspot regions. As described above, the resistance of pathogenic Acinetobacter species to broad-spectrum antibiotics has received increased attention; thus, studies examining Acinetobacter species-specific AMR mechanisms and new therapeutics for patient treatment are needed (Poirel et al., 2011). This review discusses AMR mechanisms within pathogenic Acinetobacter species and describes several studies that have used adjuvants to overcome AMR and increase the efficacy of existing antibiotics.

\section{Multidrug efflux pumps within pathogenic Acinetobacter species}

Cellular efflux-mediated extrusion, which reduces susceptibility to many antibiotics and other biocides, is a well-known mechanism in many pathogenic bacteria, including A. baumannii (Coyne et al., 2010; Beceiro et al., 2013). Bioinformatics analysis using whole genome sequencing has found that numerous multidrug transporters within the core genome of A. baumannii can eliminate many antibiotics with low specificity. A. baumannii may contain more than six different transporter superfamilies capable of actively pumping out a broad range of antimicrobial and toxic compounds from the cell (Hassan et al., 2015) (Fig. 1). Active drug transporters can be divided into two groups on the basis of their energy requirements: primary and secondary. Primary active transporters belong to the ATP-binding cassette (ABC) transporter superfamily which uses the energy from ATP hydrolysis to transport molecules across a membrane against the gradient. Secondary active transporters are ion-coupled transporters (typically $\mathrm{Na}^{+}, \mathrm{H}^{+}$, or metabolites) and include the 12-14 TM of major facilitator superfamily (MFS) efflux pump functioning as a component of a tripartite system, the $4 \mathrm{TM}$ and proton-coupled small multidrug resistance (SMR) family, the proteobacterial antimicrobial compound efflux (PACE) protein family, and the resistance-nodulation-cell division (RND) superfamily. Members of the multidrug and toxic compound extrusion (MATE) family function as drug/ sodium or proton antiporters (Fig. 1A). RND-type transporters in particular are known to play a dominant role in the MDR of many Acinetobacter species (Yoon et al., 2013).

In A. baumannii, all Ade transporters belong to the RND family and confer AMR on various antibiotics such as aminoglycosides, $\beta$-lactams, fluoroquinolones, tetracyclines, macrolides, chloramphenicol, and trimethoprim (Damier-Piolle et al., 2008; Coyne et al., 2010). Interestingly, transcriptomic analysis has also revealed the upregulation of many RND family efflux pumps under ampicillin in soil-borne A. oleivorans $\mathrm{DR} 1$; this change in the expression of many RND family proteins may be linked to ampicillin resistance (Heo et al., 2014). Each Ade system has a different affinity for each antibiotic; for example, AdeFGH cannot transport aminoglycosides (Coyne et al., 2010). Many A. baumannii clinical isolates overexpress Ade transporters, which is thought to contribute to AMR (Chu et al., 2006; Damier-Piolle et al., 2008; Coyne et al., 2010; Yoon et al., 2016). While the overexpression of Ade transporters is often beneficial to bacteria, this is not always the case; some Ade transporters, such as AdeABC, AdeFGH, and AdeIJK, can be toxic to cells when overexpressed, presumably because of changes to membrane 


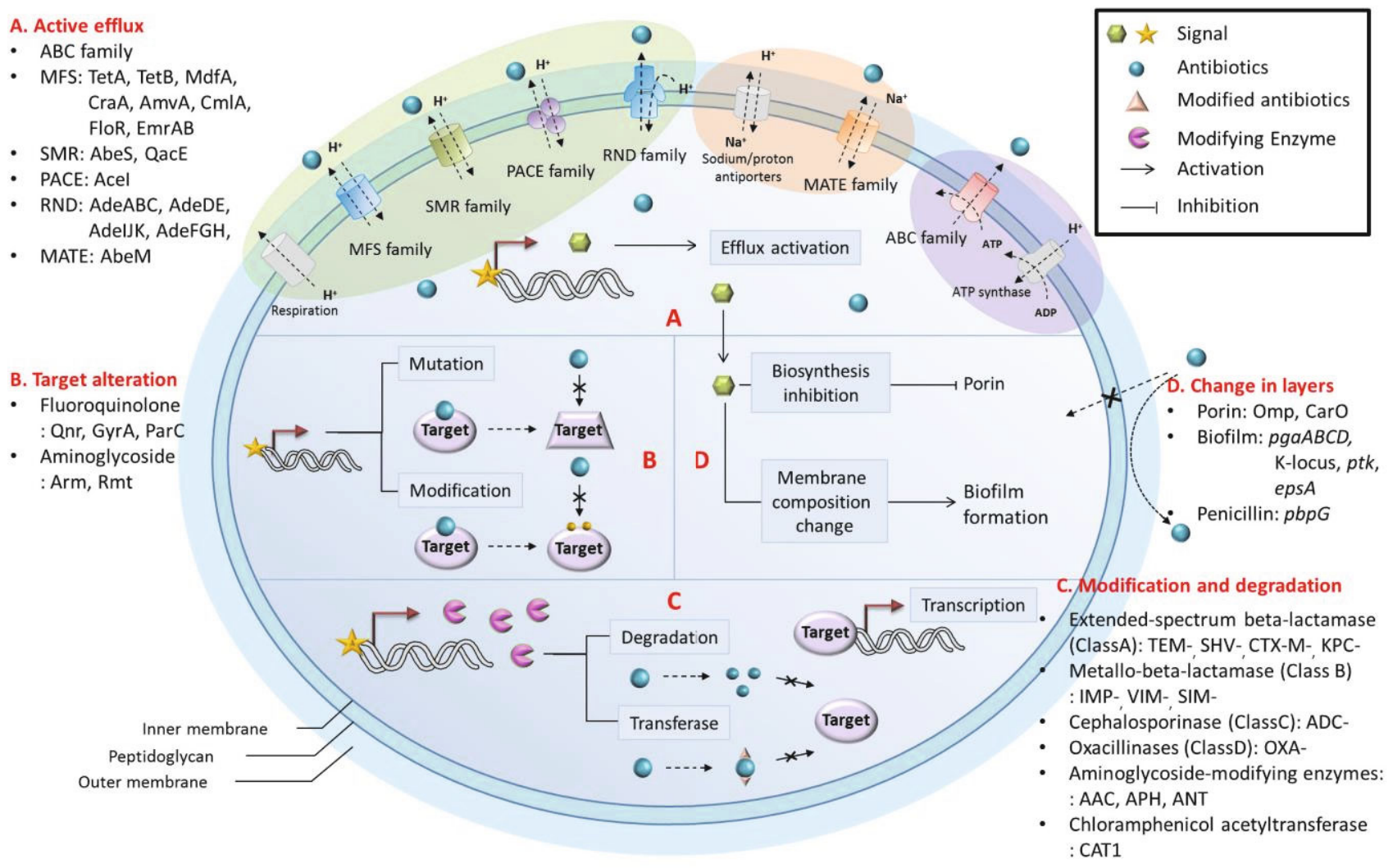

Fig. 1. Mechanisms of antibiotic resistance in Acinetobacter species. Resistance to antibiotics can be mediated by the cooperation of numerous different mechanisms. These include active efflux of the drug from the cell (A); mutation and modification of the target, thereby lowering the affinity of the drug for the target (B); modification or degradation of the drug (C); and impaired influx because of low membrane permeability (D). The large oval shape represents the bacterial cell. An electrochemical $\mathrm{H}^{+}$gradient across the cytoplasmic membrane is generated by respiration (gray). Energy from the proton-motiveforce is used to power the transport of the secondary active transport systems, such as those within the major facilitator superfamily (MFS), small multidrug resistance (SMR) family, proteobacterial antimicrobial compound efflux (PACE) family, and resistance/nodulation/division (RND) family. ATP production by ATP-synthase (gray) is also powered by the proton-motive-force, and ATP is used to power transport by the primary active transporters of the ATP-binding cassette (ABC) superfamily. Sodium/proton antiporters (gray) harness the proton-motive-force to generate an $\mathrm{Na}^{+}$gradient that powers transport by other multidrug efflux pumps, including those in the multidrug and toxic compound extrusion (MATE) family.

integrity caused by transporter overexpression (Chu et al., 2006; Damier-Piolle et al., 2008; Coyne et al., 2010). Genomic DNA Group 3 (GDG3) of Acinetobacter species expresses the AdeXYZ and AdeDE transporters, which are similar to the AdeIJK and AdeAB transporters in A. baumannii (Chau et al., 2004; Chu et al., 2006). Contribution of AdeDE to multidrug resistance was experimentally proven in the GDG3 of Acinetobacter species. Downstream sequence of AdeDE is not the ORF encoding an outer membrane protein, which implied that AdeDE might be associated with other unidentified outer membrane proteins (Chau et al., 2004).

Several MFS efflux pumps have been identified in A. baumannii, including those for tetracycline (TetA and TetB) (Ribera et al., 2003), chloramphenicol (CraA, CmlA, and FloR) (Roca et al., 2009; Coyne et al., 2011), and erythromycin (AmvA) (Rajamohan et al., 2010). Tetracycline efflux pumps, referred to as Tet proteins, are members of MFS and are often found on plasmids in pathogenic Acinetobacter strains (Nikaido, 2009). The inactivation of the CraA efflux pump, a homolog of E. coli MdfA, results in chloramphenicol susceptibility in A. baumannii (Roca et al., 2009). The homolog of the E. coli EmrAB efflux pump in A. baumannii has been shown to be involved in colistin resistance (Lin et al., 2017). The SMR family of pumps, which belong to the drug/metabolite transporter superfamily, are composed of four TM $\alpha$ helices of around 100-140 amino acids in length. In A. baumannii, the SMR family of proteins are chromosomally encoded. AbeS, which is located in the inner membrane, confers tolerance on antibiotics such as erythromycin and novobiocin as well as detergents and dyes (Srinivasan et al., 2009). The QacE protein, another well-known SMR family member, removes quaternary ammonium compounds which are important ingredients of disinfectants used in the disinfection of surfaces and medical instruments (Coyne et al., 2011). Previous research has shown that class 1 integron incidence is significantly higher for populations that are preexposed to quaternary ammonium compounds (Gaze et al., 2005). Expression analysis revealed that qacE is also upregulated in the presence of kanamycin in soil-borne A. oleivorans DR1 cells (Heo et al., 2014). Within the MATE family of proteins, AbeM is an $\mathrm{H}^{+}$-drug antiporter protein that allows for the efflux of aminoglycosides, quinolones, and chloramphenicol 
(Su et al., 2005).

The AceI (Acinetobacter chlorhexidine efflux) transporter, which is a part of the newly discovered PACE family, is proven to be important for resistance to many different biocides, antibiotics, and antimicrobial dyes (Hassan et al., 2015). Homologs of AceI are found in many proteobacteria including Pseudomonas, Bukholderia, and Vibrio species. Interestingly, AceI in A. baumannii is up-regulated 10 -fold by chlorhexidine (Hassan et al., 2015). The RND family protein $\mathrm{Ade} A \mathrm{~B}$ also appears to be involved in adaptive resistance to chlorhexidine in A. baumannii. The common laboratory strains A. baumannii AYE and ATCC 17978 contain 46 and 73 genes, respectively, that are marked as putative drug efflux pumps, suggesting that there are more novel efflux pump proteins capable of contributing to MDR in $A$. baumannii (Fournier et al., 2006; Hood et al., 2010). Also, previous research has suggested that human serum can promote the expression of unknown efflux pumps which extrude multiple antibiotics, including aminoglycosides, quinolones, and carbapenems (Jacobs et al., 2012). Understanding the role of those efflux pump proteins in modulating antibiotic tolerance is important because the development of efflux pump inhibitors can be a new way to treat infectious diseases.

\section{Mutational alteration of antibiotic targets}

The modification of antibiotic-binding sites in targets such as $16 \mathrm{~S}$ rRNA, the beta subunit of RNA polymerase, DNA gyrase, and inner and outer cell membranes is another major mechanism in pathogenic Acinetobacter species to achieve AMR (Fig. 1B). In this mechanism, genetically diverse microorganisms produced by the activity of error-prone polymerases can be selected for in the presence of a given antibiotic and then proliferate under continued antibiotic pressure. Other mechanisms for generating an adaptive bacterial population also exist. Reactive oxygen species, produced as a result of antibiotic treatment, are thought to aid in the development of adaptive populations (Dwyer et al., 2009). Intrinsic or acquired genes that encode proteins capable of modifying a drug target can also produce adaptive populations in the presence of antibiotics (Blair et al., 2015). Resistance to aminoglycoside is often conferred by aminoglycoside-modifying enzymes, including acetyltransferases, nucleotidyl transferases and phosphotransferases (Karthikeyan et al., 2010). Many genes encoding $16 \mathrm{~S}$ rRNA methylases, such as armA, rmtA-rmt $H$, and $n p m$, have been reported in Acinetobacter species (Karthikeyan et al., 2010). Of these, only $\operatorname{armA}$ is routinely associated with clinically isolated Acinetobacter species (Yu et al., 2007). Notably, the ArmA protein found in Acinetobacter is identical to that found in Salmonella enterica. Interestingly, the armA in some A. baumannii species is present on plasmids between tnpU and $\operatorname{tnpD}$ as part of the composite Tn1548 transposon (Karah et al., 2016). RmtB was also found to be present in some clinical A. baumannii strains (Beceiro et al., 2013). Because rmt genes have only recently been reported, the presence of other $r m t$ genes in pathogenic Acinetobacter strains should be investigated.

Mutations in gyrA and parC, which encode DNA gyrase and topoisomerase IV, respectively, decrease ciprofloxacin susceptibility (Vila et al., 1997). Many high-frequency heritable gyrA mutations have been observed to occur not only in pathogens but also in the soil Acinetobacter strain (Kim et al., 2013). Enzymes such as DNA gyrase and type IV topoisomerase can be protected by Qnr proteins (QnrA-D, QnrS, and QnrVC), although few studies have examined these AAC- or Qnr-based resistance mechanisms (Tran and Jacoby, 2002; Doi et al., 2004; Jiang et al., 2014). Colistin resistance occurs when lipopolysaccharide (LPS) is altered due to $\mathrm{mu}$ tations in the pmrAB two-component system and $l_{p} x A C D$ lipid A biosynthesis genes. PmrB-regulated NaxD modifies lipid A by deacetylating $\mathrm{N}$-acetylgalactosamine to galatosamine, which affects surface charge, thus lowering the affinity for cationic polymyxin antibiotics in A. baumanii (Chin et al., 2015). Many clinical isolates of Acinetobacter species contain mutations in lpxACD, which lead to the loss of LPS production or a reduction in LPS net charge (Beceiro et al., 2013; Chin et al., 2015).

\section{Antibiotic modification and degradation}

The enzymatic modification of antibiotics has been extensively reported for cases of AMR in Acinetobacter. Several mechanisms are involved, including $\beta$-lactam hydrolysis by different $\beta$-lactamases and antibiotic modification by nucleotidyltransferases, acetyltransferases, and phosphotransferases (Fig. 1C) (Rodríguez-Martínez et al., 2010). $\beta$-Lactamases that degrade carbapenems, cephalosporin, and monobactam are encoded on the chromosome and on plasmids. $\beta$-Lactamases are grouped into four classes based on their amino acid sequences (A, B, C, and D) (Rodríguez-Martínez et al., 2010). Class A $\beta$-lactamases include major extended-spectrum $\beta$-lactamases (ESBLs), such as TEM-1, TEM-2, or SHV-1, which possess a serine residue at the active site and are generally inhibited by the common $\beta$-lactamase inhibitor clavulanic acid. They are derived from narrow-spectrum betalactamases through mutations that alter the amino acid configuration around the enzyme active site (Naas et al., 2011; Bajpai et al., 2017). Clinical isolates of A. baumannii contain many different ESBLs, including SHV-12, TEM-116, CTXM-2, KPC-2 (Klebsiella pneumoniae carbapenemase-2), GES1, and VEB-1 (Naas et al., 2011; Farajnia et al., 2013).

The occurrence of class B $\beta$-lactamases, referred to as metallo- $\beta$-lactamases (MBLs), has increased with the increasing global emergence of AMR A. baumannii (Anwar et al., 2016). Four MBL enzymes have been reported to be expressed in A. baumannii: imipenemase (IMP), Verona imipenemase (VIM), Seoul imipenemase (SIM), and New Delhi metallo$\beta$-lactamase (NDM-1-type) (Higgins et al., 2010). Unlike class $A$ and class D $\beta$-lactamases, MBLs contain a metal ion, typically zinc, in the active site (Anwar et al., 2016). IMP-1, -2, $-4,-5,-6,-11$, and -55 , VIM-2, SIM-1, and NDM-1 and -2 MBLs are commonly found as part of a class 1 integron in A. baumannii (Karthikeyan et al., 2010; Naas et al., 2011; Roca et al., 2012; Azizi et al., 2016; Chatterjee et al., 2016). NDM-1 has been detected in many other pathogenic Acinetobacter species, including A. johnsonnii, A. pitti, A calcoaceticus, A. lwoffii, A. nosocomialis, A. junni, A. variabilis, and 
A. haemolyticus (Chatterjee et al., 2016; Pagano et al., 2016). Class $C \beta$-lactamases, including $a m p C$, have been found in the chromosomal DNA of certain Acinetobacter species and may represent a distinct family or Acinetobacter-derived cephalosporinases (ADCs) (Tian et al., 2011). To date, 16 bla $a_{\mathrm{ADC}}$ genes have been reported in A. baumannii: ADC-1, -3, -4, $-6,-7,-11,-26,-30,-33,-56,-57,-68,-73,-76,-77$, and -81 (Rodríguez-Martínez et al., 2010; Tian et al., 2011; Karah et al., 2016). These ADCs are commonly observed in clinical isolates. The $\beta$-lactamase genes encoded by these class $\mathrm{C}$ cephalosporinases can hydrolyze narrow-spectrum and extended-spectrum cephalosporins, but not cefepime and carbapenems. ADC-33 and ADC-56 are an exception, however, as these can hydrolyze ceftazidime and cefepime (Rodríguez-Martínez et al., 2010; Tian et al., 2011).

Class D oxacillin-hydrolyzing $\beta$-lactamases (OXA $\beta$-lactamases) are often present on plasmids and confer resistance to penicillins; however, some OXAs can also hydrolyze cephalosporins (Benjamin and Sevastian, 2014; Liao et al., 2015). Clinical isolates of carbapenem-resistant $A$. baumannii express the main oxacillinases (OXA-23, OXA-40, and OXA58; Benjamin and Sevastian, 2014). Interestingly, the outer membrane vesicles originally identified as OmpA secretion platforms include OXA-24 and OXA-58, which might contribute to increased antibiotic resistance and virulence in A. baumannii (Liao et al., 2015; Weber et al., 2017) (Fig. 1D). Other oxacillinases, such as OXA-51, have been shown to be chromosomally encoded in some A. baumannii strains (Benjamin and Sevastian, 2014). Several OXA-23-like genes (OXA-23, -24, -102, -103, -105, -133, and -134) and OXA-51/ 69-like genes have been found to be encoded in the chromosomes of $A$. radioresistens and A. baumannii isolates, respectively (Boo and Crowley, 2009; Poirel et al., 2011). Their presence in different Acinetobacter species indicates that OXA $\beta$-lactamases are essential components in the genetic makeup of the Acinetobacter genus (Benjamin and Sevastian, 2014). Outbreaks of A. baumannii harboring OXA $\beta$-lactamases have been reported worldwide, reflecting the rapid dissemination of these particular $\beta$-lactamases (Benjamin and Sevastian, 2014; Liao et al., 2015).

Aminoglycoside-modifying enzymes (nucleotidyltransferases, acetyltransferases, and/or phosphotransferases) have also been detected in most aminoglycoside-resistant clinical Acinetobacter species (Doi et al., 2004; Atasoy et al., 2015). Genes encoding aminoglycoside-modifying enzymes can be

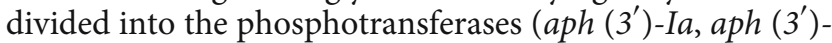
$\left.V I a, a p h\left(3^{\prime}\right)-I I\right)$, acetyltransferases (aac (3)-Ia, aac (3)-IIa, $a a c\left(6^{\prime}\right)-I b$, aac $\left(6^{\prime}\right)-I h$, aac (6')-Iad, aac (6')-Im, and $a a c$ $\left.\left(6^{\prime}\right)-I I\right)$, and nucleotidyltransferases (ant ( $\left.2^{\prime \prime}\right)-I a$, ant $\left(3^{\prime \prime}\right)$ $I a$, and ant (3")-Id) (Atasoy et al., 2015; Stogios et al., 2017). In some Acinetobacter species, quinolone drugs can be modified by acetyltransferases (AAC), commonly AAC $\left(6^{\prime}\right)$-Ibcr. Although the involvement of chloramphenicol acetyltransferase (CAT) in AMR in Acinetobacter has not been fully explained, most CAT genes have been found either chromosomally or on plasmids in clinical Acinetobacter isolates such as catI of $A$. baumannii in Tn2670; cat of $A$. calcoaceticus in Tn2670-like transposon; and catB3 of A. baumannii in chromosomes (Schwarz et al., 2004).

\section{Non-enzymatic antibiotic resistance}

Non-enzymatic AMR to antibiotics is linked to biofilm formation, changes in the affinity or composition of the cell membrane, and decreased expression of influx proteins such as porins (Fig. 1). The common structural components of most microbial biofilms are exopolysaccharides, proteins, and nucleic acids. Biofilm formation on biotic or abiotic surfaces play an important role in the ability of AMR microorganisms to persist in the environment by reducing the effective concentration of antibacterial compounds. Surface colonization of hospital equipment and medical devices by pathogenic Acinetobacter species can cause nosocomial infections (Djeribi et al., 2012). On polystyrene, a medically relevant surface, the production of pili by clinical strains is essential for biofilm formation. Csu proteins are responsible for type I pilus production, which has proven to be important for biofilm formation and virulence in Acinetobacter species. The role of pili produced by the $c s u A / B A B C D E$ six open reading frames in biofilm formation on glass and polystyrene has been reported in A. baumannii ATCC19606 strain (Tomaras et al., 2003).

For an antibacterial drug to be effective against Gram-negative bacteria, the antibiotic must penetrate the extracellular biofilm matrix, whose main components are acidic exopolysaccharides (EPSs). Poly- $\mathrm{N}$-acetylglucosamine (PNAG), which is produced in a biofilm-dependent manner, is synthesized from the pgaABCD locus and is an important EPS component for emerging pathogens such as A. baumannii (Choi et al., 2009) (Fig. 1D). However, few studies have examined the regulation and synthesis of PNAG in Acinetobacter. In addition, the detailed mechanisms by which secondary messengers such as ppGpp and c-di-GMP are linked to biofilm formation remain undefined for A. baumannii. Transcriptomic and enzymatic studies have suggested that PNAG production under static conditions is specific to pellicle formation (Nait Chabane et al., 2014). Another study, using a soil Acinetobacter strain, showed that oxidative stress causes the pga $\mathrm{ABCD}$ locus to become active, resulting in increased biofilm formation (Jang et al., 2016). However, the link between PNAG and resistance to various antibiotics remains unclear. In addition, the production of other defensive surface polysaccharides, referred to as K-locus polysaccharides, is known to protect against killing by host serum and increases bacterial virulence in animal models (Geisinger and Isberg, 2015). This K-locus-dependent capsule production, which occurs upon exposure to antibiotics, is controlled by the two-component regulatory system, bfmRS (Geisinger and Isberg, 2015). $\mathrm{BfmR}$ is negatively regulated via phosphorylation by $\mathrm{BfmS}$. When the phosphoregulation of $\mathrm{BfmR}$ is relieved under antibiotic stress, K-locus expression increases (Geisinger and Isberg, 2015). The capsular polysaccharide appears to play an important role in protecting bacteria and is a critical virulence factor that enables immune evasion (Wong et al., 2017), though how the K-locus contributes to intrinsic antibiotic resistance is poorly understood. The outer membrane also offers a much higher level of defense against antimicrobial agents because of its LPS-coated surface, which significantly limits the diffusion of many compounds, thereby lowering its permeability for hydrophobic agents (Nikaido, 2003). 
Table 2. Antibiotic and non-antibiotic combination therapy producing synergistic effects against pathogenic Acinetobacter since 2010

\begin{tabular}{|c|c|c|c|c|}
\hline Name & Combination antibiotics & $\begin{array}{l}\text { Type of } \\
\text { infection }\end{array}$ & Methods & References \\
\hline \multicolumn{5}{|l|}{ Antibiotics } \\
\hline \multirow[t]{13}{*}{ Colistin } & chloramphenicol & XDR & in vitro & Wei and Yang (2017) \\
\hline & rifampicin, meropenem, azithromycin, doxycycline & MDR & in vitro & $\begin{array}{l}\text { Timurkaynak et al. (2006), } \\
\text { Tangden et al. (2017) }\end{array}$ \\
\hline & rifampicin & CI, CR & in vivo (patients) & $\begin{array}{l}\text { Durante-Mangoni et al. (2013), } \\
\text { Aydemir } \text { et al. (2013) }\end{array}$ \\
\hline & rifampin, teicoplanin & $\mathrm{CO}$ & in vitro & Bae et al. (2016) \\
\hline & carbapenem & XDR & in vivo (patients) & Shields et al. (2012) \\
\hline & $\begin{array}{l}\text { meropenem, tigecycline, fosfomycin, fusidic acid, } \\
\text { rifampin, sulbactam }\end{array}$ & $\mathrm{XDR}$ & in vivo (mouse) & Fan et al. (2016) \\
\hline & doripenem & $\mathrm{XDR}$ & in vitro & $\begin{array}{l}\text { Shields et al. (2011) } \\
\text { Maifiah et al. (2017) }\end{array}$ \\
\hline & $\begin{array}{l}\text { carbapenem, piperacillin-tazobactam, sulbactam or } \\
\text { other }\end{array}$ & MDR & in vivo (patients) & Falagas et al. (2010) \\
\hline & vancomycin & MDR & in vitro & Gordon et al. (2010) \\
\hline & fosfomycin & $\mathrm{CI}$ & in vivo (patients) & Petrosillo et al. (2014) \\
\hline & carbapenem, sulbactam, tigecycline or other & $\mathrm{XDR}$ & in vivo (patients) & Batirel et al. (2014) \\
\hline & teicoplanin, vancomycin & MDR & in vitro & Hornsey and Wareham (2011) \\
\hline & trimethoprim-sulfamethoxazole & $\mathrm{CR}$ & in vitro & Nepka et al. (2016) \\
\hline \multirow[t]{5}{*}{ Polymyxin B } & doripenem and/or rifampin & CR & in vitro & Urban et al. (2010) \\
\hline & minocycline & $\mathrm{CI}$ & in vivo (mouse) & Bowers et al. (2015) \\
\hline & rifampicin, tigecycline & CR & in vitro & Lim et al. (2011) \\
\hline & tigecycline & CR & in vitro & Hagihara et al. (2014) \\
\hline & minocycline, fosfomycin & MDR & in vitro & Zhang et al. (2013) \\
\hline \multirow[t]{2}{*}{ Meropenem } & rifampicin & MDR & in vitro & Sun et al. (2014) \\
\hline & novel monosulfactam BAL30072 & $\mathrm{CR}$ & in vitro & Hornsey et al. (2013) \\
\hline \multirow[t]{2}{*}{ Doripenem } & levofloxacin, amikacin, colistin & $\mathrm{CI}$ & in vitro & Pankuch et al. (2010) \\
\hline & sulbactam, amikacin, colistin, tigecycline & $\mathrm{CR}$ & In vitro & Dinc et al. (2015) \\
\hline \multirow[t]{4}{*}{ Minocycline } & amikacin, rifampicin & MDR & in vitro & He et al. (2015) \\
\hline & rifampicin, colistin, imipenem & MDR & in vitro & Rodríguez et al. (2015) \\
\hline & meropenem & XDR & in vitro & Liang et al. (2011) \\
\hline & colistin & MR & in virto & Yang et al. (2016) \\
\hline \multirow[t]{5}{*}{ Tigecycline } & $\begin{array}{l}\text { ceftazidime, ceftriaxone, piperacillin/tazobactam, } \\
\text { carbapenem }\end{array}$ & MDR & in vivo (patients) & Lee et al. (2013) \\
\hline & amikacin, colistin & TR & in vitro & Li et al. (2017) \\
\hline & Meropenem, ampicillin, sulbactam & $\mathrm{CI}(\mathrm{PR})$ & in vitro & Lenhard et al. (2017) \\
\hline & colistin & $\mathrm{XDR}, \mathrm{CI}$ & in vitro & $\begin{array}{l}\text { Yilmaz et al. (2015), } \\
\text { Cai et al. (2017) }\end{array}$ \\
\hline & imipenem, sulbactam, colistin & MDR & in vitro & Pachon-Ibanez et al. (2010) \\
\hline \multirow[t]{4}{*}{ Sulbactam } & colistin (tigecycline shows a weak effect) & $\mathrm{CR}$ & in vivo (mouse) & Dinc et al. (2013) \\
\hline & ampicillin, carbapenem, cefoperazone & CI & in vivo (patients) & Chu et al. (2013) \\
\hline & $\begin{array}{l}\text { meropenem, ciprofloxacin, amikacin, tigecycline, } \\
\text { colistin }\end{array}$ & $\mathrm{CR}$ & in vitro & Temocin et al. (2015) \\
\hline & colistin & MDR, XDR & in vivo (patients) & Yilmaz et al. (2015) \\
\hline \multicolumn{5}{|l|}{ Non-antibiotics } \\
\hline Polyamine & aztreonam & CR & in vitro & Malone and Kwon (2013) \\
\hline $\begin{array}{l}\text { Epigallocatechin-3-Gallate } \\
\text { (EGCG) }\end{array}$ & $\begin{array}{l}\text { meropenem, carbenicillin, chloramphenicol, } \\
\text { tetracycline }\end{array}$ & CI & in vitro & Lee et al. (2017) \\
\hline Essential oils & iprofloxacin, gentamicin, polymyxin B & MDR & in vitro & Knezevic et al. (2016) \\
\hline Essential oils & polymixin $\mathrm{B}$, ciprofloxacine & MDR & in vitro & Aleksic et al. (2014) \\
\hline $\begin{array}{l}\text { Essential oils (Aniba rosaeodora } \\
\text { and Pelargonium graveolens) }\end{array}$ & gentamicin & RS & in vitro & Rosato et al. (2010) \\
\hline Coriander oil & $\begin{array}{l}\text { ciprofloxacin, gentamicin, chloramphenicol, } \\
\text { tetracycline }\end{array}$ & RS & in vitro & Duarte et al. (2012) \\
\hline $\begin{array}{l}\text { Carvacrol (oregano oil), eugenol } \\
\text { (clove oil), and cinnamaldehyde } \\
\text { (cinnamon oil). }\end{array}$ & doxycycline & CI & in vitro & Valcourt et al. (2016) \\
\hline $\begin{array}{l}\text { Holarrhena antidysenterica } \\
\text { extracts }\end{array}$ & rifampicin, cefazolin & $\mathrm{XDR}, \mathrm{MDR}$ & in vitro & Chusri et al. (2014a) \\
\hline
\end{tabular}


Table 2. Continued

\begin{tabular}{|c|c|c|c|c|}
\hline Name & Combination antibiotics & $\begin{array}{c}\text { Type of } \\
\text { infection }\end{array}$ & Methods & References \\
\hline Apocynaceae extracts & rifampicin, cefazolin & XDR, MDR & in vitro & Chusri et al. (2014b) \\
\hline \multirow[t]{4}{*}{ Silver nanoparticles (AgNPs) } & polymixin $\mathrm{B}$, rifampicin & $\mathrm{CR}$ & in vivo (mouse) & Wan et al. (2016) \\
\hline & oregano essential oil ${ }^{*}$ & MDR & in vitro & Scandorieiro et al. (2016) \\
\hline & piperacillin, eryth-romycin & MDR & in vitro & Ghosh et al. (2012) \\
\hline & chitosan acetate ${ }^{*}$ & MDR & in vivo (mouse) & Huang et al. (2011) \\
\hline $\begin{array}{l}\text { Zinc oxide nanoparticles } \\
\text { (ZnO-NPs) }\end{array}$ & ciprofloxacin and ceftazidime & MDR & in vitro & Ghasemi and Jalal (2016) \\
\hline Nanoscaled titania (nano $\mathrm{TiO} 2$ ) & $\begin{array}{l}\text { new isomeric carborane derivatives (a pair of } \\
\text { geometrical isomers ferrocene-carborane derivatives) }\end{array}$ & MDR & in vitro & Li et al. (2013) \\
\hline Light $\left(635 \mathrm{~nm}, 600 \mathrm{~J} / \mathrm{cm}^{2}\right)$ & chitosan & RS & in vitro & Tsai et al. (2011) \\
\hline $\begin{array}{l}\text { Light }(400-1,000 \mathrm{~nm}, \\
\left.20,000-40,000 \mathrm{~J} / \mathrm{cm}^{2}\right)\end{array}$ & colistin & CR & in vitro & Boluki et al. (2017) \\
\hline$\varphi \mathrm{km} 18 \mathrm{p}$ & $\varphi T Z 1$ and $\varphi 314$ & XDR & in vitro & Shen et al. (2012) \\
\hline LysABP-01 from ØАВР-01 & colistin & RS & in vitro & Thummeepak et al. (2016) \\
\hline Artilysins & polycationic nonapeptide & $\mathrm{CI}$ & in vitro & Briers et al. (2014) \\
\hline
\end{tabular}

* Double combination between non-antibiotic compounds.

MR, meropenem-resistant; CO, Colistin-Resistant; IR, imipenem-resistant; CI, Clinical isolate; CR, Carbapenem-resistant; TR, Tigecycline-resistant; PR, Polymyxin B-resistant; MDR, Multidrug-resistant; XDR, Extremely drug-resistant

Porins are $\beta$-barrel membrane proteins that cross cell membranes and act as pores through which molecules such as nutrients, toxins, and antibiotics can pass. The alteration, modification, and reduction of the expression of porins are related to antibiotic resistance (Sugawara and Nikaido, 2012). Using in vivo protein interaction network analysis, a recent report on outer membrane porins from a clinical isolate of $A$. baumannii revealed that a significant amounts of oxacilinases interact with outer membrane porins including OmpA, CarO, and OmpW (Wu et al., 2016). The A. baumannii OmpA protein was recently reported to be associated with resistance to cephalothin and cephaloridine in A. baumannii (Sugawara and Nikaido, 2012). It has been also reported that the inactivation of CarO causes increased resistance to carbapenems in A. baumannii strains (Hood et al., 2010). The overall results indicate that CarO participates in the selective uptake of L-ornithine, carbapenems, and other basic amino acids in A. baumannii (Mussi et al., 2007). The interaction of these functional porins with protective enzymes can be understood as a general advantageous strategy against antibacterial compounds (Wu et al., 2016).

In vitro-selected mutants resistant to cefoxitin, cefoperazone, or ceftazidime also exhibit reduced expression of porins and changes in PBP expression and/or the affinity for $\beta$-lactams in A. calcoaceticus (Obara and Nakae, 1991). Similarly, proteome analysis was performed in an imipenem-resistant $A$. baumannii strain under imipenem treatment conditions (Yun et al.,. 2011). When imipenem-resistant $A$. baumannii was treated with imipenem, the induction of RND family transporters (AdeABC and AdeJIK), a protein kinase (BfmS), and PBPs, as well as reduction of the OMPs OmpA and OmpW was observed. These results suggest that these mechanisms together contribute to imipenem resistance in A. baumannii.

\section{Treatment of Acinetobacter infections}

To treat infection of MDR pathogens, a series of new qui- nolones such as levofloxacin, ciprofloxacin, moxifloxacin, ofloxacin, and gemifloxacin have been developed as antibacterial drugs (Jiang et al., 2014; Blair et al., 2015). However, resistance to new antibiotics arises quickly. Thus, new therapeutic methods are needed to combat MDR pathogens. The development of a new single drug is costly and timeconsuming, thus studies have focused on combination treatments. Despite the prevalence of antibiotic resistance, the current body of research is too small to allow clinicians to select an optimal treatment for $A$. baumannii infections. To combat serious A. baumannii-associated infections, various trials have been conducted using antibiotics, bacteriophages, plant extracts, and nanoparticles (Djeribi et al., 2012) (Table 2). In vitro and in vivo studies have shown that combinations of drugs may be synergic and highly bactericidal when used against clinical isolates of drug-resistant $A$. baumannii (Table 2). Such synergic combinations typically include two or three different classes of antibiotics. It has already been shown in many studies that the effect of antibiotics is increased by the addition of other antimicrobial agents such as chimeric peptides (Gopal et al., 2014). Advantages of combination therapies include a broad spectrum of antibacterial coverage, synergistic effects between different antimicrobial compounds, and the prevention of resistance (Lutsar et al., 2014). To summarize the extensive in vitro and in vivo information shown in Table 2, recent combination experiments have primarily focused on polymyxins, sulbactam, tigecycline, and rifampin or carbapenems.

Polymyxins have been successfully used as an aerosol to treat ventilator-associated pneumonia caused by $A$. baumannii, with lower nephrotoxicity than predicted (Urban et al., 2001; Gales et al., 2006). Polymyxin E (colistin) is present in two forms, colistin sulfate for oral and topical use and colistin sulfomethate sodium for parenteral use, with the latter being a non-active prodrug used for parenteral administration because of its lower toxicity. Polymyxins are cationic polypeptides that interact with LPS molecules in the outer cell membranes of Gram-negative bacteria. A. baumannii strains 
are susceptible to polymyxins, which are toxic to humans, and therefore therapies that combine polymyxins with other antibiotics to decrease their side effects by reducing the dose have already been used to treat A. baumannii infections in humans (Falagas et al., 2010). The in vitro synergism of colistin with carbapenem, rifampicin, tigecycline and other antibiotics against $A$. baumannii has been reported (Table 2). In addition, the in vivo results reported for solid organ transplant recipients who were colonized by or infected with extremely drug-resistant (XDR) A. baumannii suggest that a combination of colistin and carbapenem improves clinical responses and survival compared to other therapies such as colistin-tigecycline and may also limit the emergence of colistin resistance (Shields et al., 2012) (Table 2). The combination colisin and rifampicin in the treatment of ventilator-associated pneumonia has also demonstrated improved clinical outcomes (Aydemir et al., 2013) (Table 2). However, 30-day mortality was not reduced by the addition of rifampicin to colistin despite the increased mortality rates of A. baumannii cells (Durante-Mangoni et al., 2013) (Table 2).

Of the $\beta$-lactamase inhibitors, sulbactam has shown the strongest intrinsic bactericidal activity against $A$. baumannii isolates. The results of clinical investigations have documented the efficacy of sulbactam in mild-to-severe A. baumannii infections, and sulbactam has been successfully used to treat infections associated with MDR A. baumannii such as meningitis, ventilator-associated pneumonia, and catheter-related bacteremia (Temocin et al., 2015). In most patients, sulbactam has been used in combination with ampicillin and carbapenems but its usefulness is increasingly compromised by bacterial resistance. A recent study on the use of tigecycline against XDR A. baumannii infections analyzed 120 patients who were treated with imipenem and sulbactam (Lee et al., 2013) (Table 2). Even if the dosage is reduced in combination therapies, the prolonged use of antibiotics can disturb the normal gastrointestinal microflora, reducing the natural defense mechanisms provided by the microbial exosystem in the colon and making the host susceptible to infection by symbiotic microorganisms or nosocomial pathogens (Rafii et al., 2008). Despite conflicting evidence of their efficiency due to each toxicity, complex antibiotics with different mechanisms of action are commonly used in severe infections.

\section{Other possible approaches to treat pathogenic Acinetobacter infection}

To overcome the risks inherent to the use of antibiotics, the number of other available combination treatments needs to be expanded. The extracts of plants used in traditional medicine have been used to reduce the side effects of antibiotic resistance and combination therapy (Tiwari et al., 2015). The ineffectiveness of synthetic antibiotics against drug-resistant bacteria has led to the reemergence of interest in silver and other non-antibiotics that have a long history as antibacterial agents. Oleanolic acid and ursolic acid can act synergistically in combination with aminoglycosides to increase energy and membrane permeability (Shin and Park, 2015). The antimicrobial activity of essential oils, polyamines and nanoparticles has been intensively explored, mainly in the course of developing new antimicrobial agents to overcome microbial resistance (Malone and Kwon, 2013; Wan et al., 2016; Chaib et al., 2017) (Table 2). Some compounds in plants are potently antimicrobial when used against $A$. baumannii. For examples, traditional medical herb extracts from $L$. salicaria exhibit antibacterial activity against $A$. baumannii (Guclu et al., 2014). In addition, essential oils from Syzygium aromaticum, Cinnamomum zeylanicum, and Thymus are effective as nanomedicines against multidrug-resistant $A$. baumannii (Tiwari et al., 2015). Compounds from Scutellaria baicalensis such as baicalin and baicalein have also been reported to have antibacterial properties (Tiwari et al., 2015). Studies have shown that many phenolic compounds derived from plant extracts enhance the effectiveness of synthetic antibiotics against $A$. baumannii in vitro (Tiwari et al., 2015).

The antibacterial effects of polyamines (spermine and spermidine), which are small molecular compounds with positively charged amine groups existing at millimolar levels in all living cells, against carbapenem-resistant $A$. baumannii increase significantly when used in conjunction with aztreonam (Malone and Kwon, 2013). The activity of many natural substances against $A$. baumannii has been tested, but the exact mechanisms by which certain compounds act in synergy with each other have not been defined and further research is needed. A combination therapy approach to the development of chemically modified synthetic substances based on the structure of natural active compounds derived from medicinal herbs may be a novel form of treatment for the MDR and XDR strains of A. baumannii.

Bacteriophage has been considered as an possible option for infectious disease, but research on Acinetobacter was mainly about transduction, phage typing or phage classification (Shen et al., 2012). Despite the large number of publications on phage therapy, little is known about its antibiotic combination therapy against $A$. baumanii. Recently, bacteriophages and their endolysins have been recognized as alternative therapeutic agents that can combat drug-resistant bacterial infections. For example, in A. baumannii, the synergistic antibacterial effect of $\varphi \mathrm{km} 18 \mathrm{p}$ has been only observed in combination with other phages such as $\varphi \mathrm{TZ} 1$ and $\varphi 314$ (Shen et al., 2012) (Table 2). Some Acinetobacter genus are known to sense and respond to light, which can modulate metabolic pathways including phenylacetic acid, trehalose, acetoin, and also change lipid metabolism, virulence, and resistance to antibiotics (Ramírez et al., 2015). It was revealed that light can reduce susceptibility to minocycline and tigecycline and modulate motility, biofilm formation, and virulence through the blue-light-sensing-using flavin photoreceptor BlsA (Muller et al., 2017). However, photodynamic inactivation (PDI) which uses nontoxic chemicals with harmless visible light was shown to be a promising treatment method against $A$. baumannii (Tsai et al., 2011). Several studies also suggested that effectiveness of PDI for killing Gram-negative bacteria could be promoted in the presence of some antibiotics including polymyxin B (Tsai et al., 2011). Interestingly, chitosan alone has no significant antimicrobial activity, but the PDI treatment with chitosan effectively killed many Gram-negative pathogenic bacteria. The PDI appeared to have a synergistic effect with colistin for killing a pan-drug resistant strain of A. baumannii isolated from burn patients (Boluki 
et al., 2017). To date, little is known about the PDI combination treatment antibacterial compounds against $A$. baumannii (Table 2). Antimicrobial combination therapy including the PDI appears to be a reasonable alternative in the treatment of MDR A. baumannii. However, clinical experience with combination therapy is also limited. Developing a sufficient volume of usable natural compounds may be an obstacle to the development of new medicines as the supply should be large enough to first support initial in vitro/in vivo studies.

\section{Concluding Remarks}

More than half a century has passed since the first antibiotics were introduced commercially. Microbes quickly developed drug resistance to these original antibacterial drugs. Multidrug resistance is now a worldwide problem that does not recognize international borders. Although conventional antibiotics have proven efficacious in the treatment of bacterial infections, non-antibiotics such as nanoparticles and herbal medicines may offer alternative treatment options. Because MDR is increasing, clinicians require a novel form of treatment for resistant bacteria such as A. baumannii. There is a need to develop new drugs that can potentially be used to treat infections caused by currently resistant Acinetobacter pathogens (Lutsar et al., 2014). Non-antibiotics such as silver, ethanol extracts of something such as plants, and tea tree oil are currently available as topical formulations (Dai et al., 2010), and these products may in the future play a role in reducing the toxicity of antibiotics to human organs and other serious side effects.

Some herbal extracts and compounds, such as those from green tea, are known to be less toxic to native gastrointestinal flora (Cabrera et al., 2006). If a synergistic combination of compounds removes an infection more efficiently, the duration of treatment may be shortened and associated antibiotic toxicity may be reduced. Thus, the more efficient killing of microbes may decrease the risk of progressively increasing antibiotic resistance. In the future, a detailed understanding of both the conditions under which resistance occurs and the synergy arising from various combinations of promising compounds will help clinicians to develop more efficient dosing regimens that minimize resistance to current and new antibiotics. As interest in antibiotic resistance increases, members of the genus Acinetobacter, which are ubiquitous in hospitals and natural environments, have emerged as model bacteria to study of sharing genes horizontally through conjugation, transformation, and transduction (Jung and Park, 2015). The transfer of virulence genes from a nonpathogenic bacterial strain to a pathogenic strain may result in the emergence of potentially powerful new pathogens which can gain antibiotic resistance from environmental bacteria (Beceiro et al., 2013). The increase in MDR and pan-drug resistant A. baumannii is a growing concern, and the production of new antibiotics, including host-defense peptides, is therefore urgently required. The molecular mechanisms of drug resistance need to be described so that this information can be used in the development of new drugs and subsequent clinical use.

\section{Acknowledgements}

This work was supported by a National Research Foundation of Korea (NRF) grant to WP funded by the Korean government (MSIP) (No. NRF-2017R1A2B4005838).

\section{References}

Aleksic, V., Mimica-Dukic, N., Simin, N., Nedeljkovic, N.S., and Knezevic, P. 2014. Synergistic effect of Myrtus communis L. essential oils and conventional antibiotics against multi-drug resistant Acinetobacter baumannii wound isolates. Phytomedicine 21, 16661674.

Anwar, M., Ejaz, H., Zafar, A., and Hamid, H. 2016. Phenotypic detection of metallo-beta-lactamases in carbapenem resistant Acinetobacter baumannii isolated from pediatric patients in Pakistan. J. Pathog. 2016, 8603964.

Atasoy, A.R., Ciftci, I.H., and Petek, M. 2015. Modifying enzymes related aminoglycoside: analyses of resistant Acinetobacter isolates. Int. J. Clin. Exp. Med. 8, 2874-2880.

Aydemir, H., Akduman, D., Piskin, N., Comert, F., Horuz, E., Terzi, A., Kokturk, F., Ornek, T., and Celebi, G. 2013. Colistin vs. the combination of colistin and rifampicin for the treatment of carbapenem-resistant Acinetobacter baumannii ventilator-associated pneumonia. Epidemiol. Infect. 141, 1214-1222.

Azizi, O., Shakibaie, M.R., Badmasti, F., Modarresi, F., Ramazanzadeh, R., Mansouri, S., and Shahcheraghi, F. 2016. Class 1 integrons in non-clonal multidrug-resistant Acinetobacter baumannii from Iran, description of the new blaIMP-55 allele in In1243. J. Med. Microbiol. 65, 928-936.

Bae, S., Kim, M.C., Park, S.J., Kim, H.S., Sung, H., Kim, M.N., Kim, S.H., Lee, S.O., Choi, S.H., Woo, J.H., et al. 2016. In vitro synergistic activity of antimicrobial agents in combination against clinical isolates of colistin-resistant Acinetobacter baumannii. Antimicrob. Agents Chemother. 60, 6774-6779.

Bajpai, T., Pandey, M., Varma, M., and Bhatambare, G.S. 2017. Prevalence of TEM, SHV, and CTX-M Beta-Lactamase genes in the urinary isolates of a tertiary care hospital. Avicenna. J. Med. 7, $12-16$.

Batirel, A., Balkan, Ii, Karabay, O., Agalar, C., Akalin, S., Alici, O., Alp, E., Altay, F.A., Altin, N., Arslan, F., et al. 2014. Comparison of colistin-carbapenem, colistin-sulbactam, and colistin plus other antibacterial agents for the treatment of extremely drug-resistant Acinetobacter baumannii bloodstream infections. Eur. J. Clin. Microbiol. Infect. Dis. 33, 1311-1322.

Beceiro, A., Tomás, M., and Bou, G. 2013. Antimicrobial resistance and virulence: a successful or deleterious association in the bacterial world? Clin. Microbiol. Rev. 26, 185-230.

Benjamin, A. and Sebastian, G.B. 2014. OXA $\beta$-lactamases. Clin. Microbiol. Rev. 27, 241-263.

Blair, J.M., Webber, M.A., Baylay, A.J., Ogbolu, D.O., and Piddock, L.J. 2015. Molecular mechanisms of antibiotic resistance. Nat. Rev. Microbiol. 13, 42-51.

Boluki, E., Kazemian, H., Peeridogaheh, H., Alikhani, M.Y., Shahabi, S., Beytollahi, L., and Ghorbanzadeh, R. 2017. Antimicrobial activity of photodynamic therapy in combination with colistin against a pan-drug resistant Acinetobacter baumannii isolated from burn patient. Photodiagnosis Photodyn. Ther. 18, 1-5.

Boo, T.W. and Crowley, B. 2009. Detection of blaOXA-58 and blaOXA-23-like genes in carbapenem-susceptible Acinetobacter clinical isolates: should we be concerned? J. Med. Microbiol. 58, 839-841.

Bowers, D.R., Cao, H., Zhou, J., Ledesma, K.R., Sun, D., Lomovskaya, O., and Tam, V.H. 2015. Assessment of minocycline and polymyxin B combination against Acinetobacter baumannii. Anti- 
microb. Agents Chemother. 59, 2720-2725.

Briers, Y., Walmagh, M., Van Puyenbroeck, V., Cornelissen, A., Cenens, W., Aertsen, A., Oliveira, H., Azeredo, J., Verween, G., Pirnay, J.P., et al. 2014. Engineered endolysin-based "Artilysins" to combat multidrug-resistant Gram-negative pathogens. MBio 5, e01379-14.

Cabrera, C., Artacho, R., and Gimenez, R. 2006. Beneficial effects of green tea--a review. J. Am. Coll. Nutr. 25, 79-99.

Cai, X., Yang, Z., Dai, J., Chen, K., Zhang, L., Ni, W., Wei, C., and Cui, J. 2017. Pharmacodynamics of tigecycline alone and in combination with colistin against clinical isolates of multidrug-resistant Acinetobacter baumannii in an in vitro pharmacodynamic model. Int. J. Antimicrob. Agents 49, 609-616.

Chaib, F., Allali, H., Bennaceur, M., and Flamini, G. 2017. Chemical composition and antimicrobial activity of essential oils from the aerial parts of Asteriscus graveolens (FORSSK.) LESS. and Pulicaria incisa (LAM.) DC.: Two asteraceae herbs growing wild in the hoggar. Chem. Biodivers. 14, e1700092.

Chatterjee, S., Datta, S., Roy, S., Ramanan, L., Saha, A., and Viswanathan, R. 2016. Carbapenem resistance in Acinetobacter baumannii and other Acinetobacter spp. causing neonatal sepsis: focus on NDM-1 and its linkage to ISAba125. Front. Microbiol. 7, 1126.

Chau, S.L., Chu, Y.W., and Houang, E.T. 2004. Novel resistance nodulation-cell division efflux system AdeDE in Acinetobacter genomic DNA group 3. Antimicrob. Agents Chemother. 48, 40544055.

Chin, C.Y., Gregg, K.A., Napier, B.A., Ernst, R.K., and Weiss, D.S. 2015. A PmrB-regulated deacetylase required for lipid a modification and polymyxin resistance in Acinetobacter baumannii. Antimicrob. Agents Chemother. 59, 7911-7914.

Choi, A.H., Slamti, L., Avci, F.Y., Pier, G.B., and Maira-Litrán, T. 2009. The pgaABCD locus of Acinetobacter baumannii encodes the production of poly-beta-1-6-N-acetylglucosamine, which is critical for biofilm formation. J. Bacteriol. 191, 5953-5963.

Chu, Y.W., Chau, S.L., and Houang, E.T. 2006. Presence of active efflux systems AdeABC, AdeDE and AdeXYZ in different Acinetobacter genomic DNA groups. J. Med. Microbiol. 55, 477-478.

Chu, H., Zhao, L., Wang, M., Liu, Y., Gui, T., and Zhang, J. 2013. Sulbactam-based therapy for Acinetobacter baumannii infection: a systematic review and meta-analysis. Braz. J. Infect. Dis. 17, 389394.

Chusri, S., Na-Phatthalung, P., Siriyong, T., Paosen, S., and Voravuthikunchai, S.P. 2014a. Holarrhena antidysenterica as a resistance modifying agent against Acinetobacter baumannii: Its effects on bacterial outer membrane permeability and efflux pumps. Microbiol. Res. 169, 417-424.

Chusri, S., Siriyong, T., Na-Phatthalung, P., and Voravuthikunchai, S.P. 2014b. Synergistic effects of ethnomedicinal plants of Apocynaceae family and antibiotics against clinical isolates of Acinetobacter baumannii. Asian Pac. J. Trop. Med. 7, 456-461.

Coyne, S., Courvalin, P., and Périchon, B. 2011. Efflux-mediated antibiotic resistance in Acinetobacter spp. Antimicrob. Agents Chemother. 55, 947-953.

Coyne, S., Rosenfeld, N., Lambert, T., Courvalin, P., and Périchon, B. 2010. Overexpression of resistance-nodulation-cell division pump AdeFGH confers multidrug resistance in Acinetobacter baumannii. Antimicrob. Agents Chemother. 54, 4389-4393.

Dai, T., Huang, Y.Y., Sharma, S.K., Hashmi, J.T., Kurup, D.B., and Hamblin, M.R. 2010. Topical antimicrobials for burn wound infections. Recent Pat. Antiinfect. Drug Discov. 5, 124-151.

Damier-Piolle, L., Magnet, S., Brémont, S., Lambert, T., and Courvalin, P. 2008. AdeIJK, a resistance-nodulation-cell division pump effluxing multiple antibiotics in Acinetobacter baumannii. Antimicrob. Agents Chemother. 52, 557-562.

Dinc, G., Demiraslan, H., Elmali, F., Ahmed, S.S., Alp, E., and Doganay, M. 2015. Antimicrobial efficacy of doripenem and its combinations with sulbactam, amikacin, colistin, tigecycline in ex- perimental sepsis of carbapenem-resistant Acinetobacter baumannii. New Microbiol. 38, 67-73.

Dinc, G., Demiraslan, H., Elmali, F., Ahmed, S.S., Metan, G., Alp, E., and Doganay, M. 2013. Efficacy of sulbactam and its combination with imipenem, colistin and tigecycline in an experimental model of carbapenem-resistant Acinetobacter baumannii sepsis. Chemotherapy 59, 325-329.

Djeribi, R., Bouchloukh, W., Jouenne, T., and Menaa, B. 2012. Characterization of bacterial biofilms formed on urinary catheters. Am. J. Infect. Control. 40, 854-859.

Doi, Y., Wachino, J., Yamane, K., Shibata, N., Yagi, T., Shibayama, K., Kato, H., and Arakawa, Y. 2004. Spread of novel aminoglycoside resistance gene aac (6')-Iad among Acinetobacter clinical isolates in Japan. Antimicrob. Agents Chemother. 48, 2075-2080.

Duarte, A., Ferreira, S., Silva, F., and Domingues, F.C. 2012. Synergistic activity of coriander oil and conventional antibiotics against Acinetobacter baumannii. Phytomedicine 19, 236-238.

Durante-Mangoni, E., Signoriello, G., Andini, R., Mattei, A., De Cristoforo, M., Murino, P., Bassetti, M., Malacarne, P., Petrosillo, N., Galdieri, N., et al. 2013. Colistin and rifampicin compared with colistin alone for the treatment of serious infections due to extensively drug-resistant Acinetobacter baumannii: a multicenter, randomized clinical trial. Clin. Infect. Dis. 57, 349-358.

Dwyer, D.J., Kohanski, M.A., and Collins, J.J. 2009. Role of reactive oxygen species in antibiotic action and resistance. Curr. Opin. Microbiol. 12, 482-489.

Falagas, M.E., Rafailidis, P.I., Ioannidou, E., Alexiou, V.G., Matthaiou, D.K., Karageorgopoulos, D.E., Kapaskelis, A., Nikita, D., and Michalopoulos, A. 2010. Colistin therapy for microbiologically documented multidrug-resistant Gram-negative bacterial infections: a retrospective cohort study of 258 patients. Int. J. Antimicrob. Agents 35, 194-199.

Fan, B., Guan, J., Wang, X., and Cong, Y. 2016. Activity of colistin in combination with meropenem, tigecycline, fosfomycin, fusidic acid, rifampin or sulbactam against extensively drug-resistant Acinetobacter baumannii in a murine thigh-infection model. PLoS One 11, e0157757.

Farajnia, S., Azhari, F., Alikhani, M.Y., Hosseini, M.K., Peymani, A., and Sohrabi, N. 2013. Prevalence of PER and VEB type extended spectrum betalactamases among multidrug resistant Acinetobacter baumannii isolates in North-West of Iran. Iran J. Basic Med. Sci. 16, 751-755.

Fournier, P.E., Vallenet, D., Barbe, V., Audic, S., Ogata, H., Poirel, L., Richet, H., Robert, C., Mangenot, S., Abergel, C., et al. 2006. Comparative genomics of multidrug resistance in Acinetobacter baumannii. PLoS Genet. 2, e7.

Gales, A.C., Jones, R.N., and Sader, H.S. 2006. Global assessment of the antimicrobial activity of polymyxin B against 54731 clinical isolates of Gram-negative bacilli: report from the SENTRY antimicrobial surveillance programme (2001-2004). Clin. Microbiol. Infect. 12, 315-321.

Gaze, W.H., Abdouslam, N., Hawkey, P.M., and Wellington, E.M.H. 2005. Incidence of class 1 integrons in a quaternary ammonium compound-polluted environment. Antimicrob. Agents Chemother. 49, 1802-1807.

Geisinger, E. and Isberg, R.R. 2015. Antibiotic modulation of capsular exopolysaccharide and virulence in Acinetobacter baumannii. PLoS Pathog. 11, e1004691.

Ghasemi, F. and Jalal, R. 2016. Antimicrobial action of zinc oxide nanoparticles in combination with ciprofloxacin and ceftazidime against multidrug-resistant Acinetobacter baumannii. J. Glob. Antimicrob. Resist. 6, 118-122.

Ghosh, S., Patil, S., Ahire, M., Kitture, R., Kale, S., Pardesi, K., Cameotra, S.S., Bellare, J., Dhavale, D.D., Jabgunde, A., et al. 2012. Synthesis of silver nanoparticles using Dioscorea bulbifera tuber extract and evaluation of its synergistic potential in combination with antimicrobial agents. Int. J. Nanomedicine 7, 483-496. 
Gopal, R., Kim, Y.G., Lee, J.H., Lee, S.K., Chae, J.D., Son, B.K., Seo, C.H., and Park, Y. 2014. Synergistic effects and antibiofilm properties of chimeric peptides against multidrug-resistant Acinetobacter baumannii strains. Antimicrob. Agents Chemother. 58, 1622-1629.

Gordon, N.C., Png, K., and Wareham, D.W. 2010. Potent synergy and sustained bactericidal activity of a vancomycin-colistin combination versus multidrug-resistant strains of Acinetobacter baumannii. Antimicrob. Agents Chemother. 54, 5316-5322.

Guclu, E., Genc, H., Zengin, M., and Karabay, O. 2014. Antibacterial activity of Lythrum salicaria against multidrug-resistant Acinetobacter baumannii and Pseudomonas aeruginosa. Annu. Res. Rev. Biol. 4, 1099-1105.

Hagihara, M., Housman, S.T., Nicolau, D.P., and Kuti, J.L. 2014. In vitro pharmacodynamics of polymyxin $\mathrm{B}$ and tigecycline alone and in combination against carbapenem-resistant Acinetobacter baumannii. Antimicrob. Agents Chemother. 58, 874-879.

Hassan, K.A., Liu, Q., Henderson, P.J., and Paulsen, I.T. 2015. Homologs of the Acinetobacter baumannii AceI transporter represent a new family of bacterial multidrug efflux systems. MBio 6, e01982-14

He, S., He, H., Chen, Y., Chen, Y., Wang, W., and Yu, D. 2015. In vitro and in vivo analysis of antimicrobial agents alone and in combination against multi-drug resistant Acinetobacter baumannii. Front. Microbiol. 6, 507.

Heo, A., Jang, H.J., Sung, J.S., and Park, W. 2014. Global transcriptome and physiological responses of Acinetobacter oleivorans DR1 exposed to distinct classes of antibiotics. PLoS One 9, e110215.

Higgins, P.G., Dammhayn, C., Hackel, M., and Seifert, H. 2010. Global spread of carbapenem-resistant Acinetobacter baumannii. J. Antimicrob. Chemother. 65, 233-238.

Hood, M.I., Jacobs, A.C., Sayood, K., Dunman, P.M., and Skaar, E.P. 2010. Acinetobacter baumannii increases tolerance to antibiotics in response to monovalent cations. Antimicrob. Agents Chemother. 54, 1029-1041.

Hornsey, M., Phee, L., Stubbings, W., and Wareham, D.W. 2013. In vitro activity of the novel monosulfactam BAL30072 alone and in combination with meropenem versus a diverse collection of important Gram-negative pathogens. Int. J. Antimicrob. Agents 42, 343-346.

Hornsey, M. and Wareham, D.W. 2011. In vivo efficacy of glycopeptide-colistin combination therapies in a Galleria mellonella model of Acinetobacter baumannii infection. Antimicrob. Agents Chemother. 55, 3534-3537.

Huang, L., Dai, T., Xuan, Y., Tegos, G.P., and Hamblin, M.R. 2011. Synergistic combination of chitosan acetate with nanoparticle silver as a topical antimicrobial: efficacy against bacterial burn infections. Antimicrob. Agents Chemother. 55, 3432-3438.

Jacobs, A.C., Sayood, K., Olmsted, S.B., Blanchard, C.E., Hinrichs, S., Russell, D., and Dunman, P.M. 2012. Characterization of the Acinetobacter baumannii growth phase-dependent and serum responsive transcriptomes. FEMS Immunol. Med. Microbiol. 64, 403-412.

Jang, I.A., Kim, J., and Park, W. 2016. Endogenous hydrogen peroxide increases biofilm formation by inducing exopolysaccharide production in Acinetobacter oleivorans DR1. Sci. Rep. 6, 21121.

Jiang, X., Yu, T., Jiang, X., Zhang, W., Zhang, L., and Ma, J. 2014. Emergence of plasmid-mediated quinolone resistance genes in clinical isolates of Acinetobacter baumannii and Pseudomonas aeruginosa in Henan, China. Diagn. Microbiol. Infect. Dis. 79, 381-383.

Jung, J. and Park, W. 2015. Acinetobacter species as model microorganisms in environmental microbiology: current state and perspectives. Appl. Microbiol. Biotechnol. 99, 2533-2548.

Karah, N., Dwibedi, C.K., Sjostrom, K., Edquist, P., Johansson, A., Wai, S.N., and Uhlin, B.E. 2016. Novel aminoglycoside resistance transposons and transposon-derived circular forms de- tected in carbapenem-resistant Acinetobacter baumannii clinical isolates. Antimicrob. Agents Chemother. 60, 1801-1818.

Karthikeyan, K., Thirunarayan, M.A., and Krishnan, P. 2010. Coexistence of blaOXA-23 with blaNDM-1 and armA in clinical isolates of Acinetobacter baumannii from India. J. Antimicrob. Chemother. 65, 2253-2254.

Kim, J., Noh, J., and Park, W. 2013. Insight into norfloxacin resistance of Acinetobacter oleivorans DR1: target gene mutation, persister, and RNA-Seq analyses. J. Microbiol. Biotechnol. 23, 12931303.

Knezevic, P., Aleksic, V., Simin, N., Svircev, E., Petrovic, A., and Mimica-Dukic, N. 2016. Antimicrobial activity of Eucalyptus camaldulensis essential oils and their interactions with conventional antimicrobial agents against multi-drug resistant Acinetobacter baumannii. J. Ethnopharmacol. 178, 125-136.

Lee, S., Razqan, G.S., and Kwon, D.H. 2017. Antibacterial activity of epigallocatechin-3-gallate (EGCG) and its synergism with betalactam antibiotics sensitizing carbapenem-associated multidrug resistant clinical isolates of Acinetobacter baumannii. Phytomedicine 24, 49-55.

Lee, Y.T., Tsao, S.M., and Hsueh, P.R. 2013. Clinical outcomes of tigecycline alone or in combination with other antimicrobial agents for the treatment of patients with healthcare-associated multidrug-resistant Acinetobacter baumannii infections. Eur. J. Clin. Microbiol. Infect. Dis. 32, 1211-1220.

Lenhard, J.R., Thamlikitkul, V., Silveira, F.P., Garonzik, S.M., Tao, X., Forrest, A., Soo Shin, B., Kaye, K.S., Bulitta, J.B., Nation, R.L., et al. 2017. Polymyxin-resistant, carbapenem-resistant Acinetobacter baumannii is eradicated by a triple combination of agents that lack individual activity. J. Antimicrob. Chemother. 72, 14151420.

Li, S., Wu, C., Zhao, X., Jiang, H., Yan, H., and Wang, X. 2013. Synergistic antibacterial activity of new isomeric carborane derivatives through combination with nanoscaled titania. J. Biomed. Nanotechnol. 9, 393-402.

Li, J., Yang, X., Chen, L., Duan, X., and Jiang, Z. 2017. In vitro activity of various antibiotics in combination with tigecycline against Acinetobacter baumannii: A systematic review and meta-analysis. Microb. Drug. Resist. DOI: 10.1089/mdr.2016.0279.

Liang, W., Liu, X.F., Huang, J., Zhu, D.M., Li, J., and Zhang, J. 2011. Activities of colistin- and minocycline-based combinations against extensive drug resistant Acinetobacter baumannii isolates from intensive care unit patients. BMC Infect. Dis. 11, 109.

Liao, Y.T., Kuo, S.C., Chiang, M.H., Lee, Y.T., Sung, W.C., Chen, Y.H., Chen, T.L., and Fung, C.P. 2015. Acinetobacter baumannii extracellular OXA-58 is primarily and selectively released via outer membrane vesicles after Sec-dependent periplasmic translocation. Antimicrob. Agents Chemother. 59, 7346-7354.

Lim, T.P., Tan, T.Y., Lee, W., Sasikala, S., Tan, T.T., Hsu, L.Y., and Kwa, A.L. 2011. In-vitro activity of polymyxin B, rifampicin, tigecycline alone and in combination against carbapenem-resistant Acinetobacter baumannii in Singapore. PLoS One 6, e18485.

Lin, M.F., Lin, Y.Y., and Lan, C.Y. 2017. Contribution of EmrAB efflux pumps to colistin resistance in Acinetobacter baumannii. J. Microbiol. 55, 130-136.

Lutsar, I., Telling, K., and Metsvaht, T. 2014. Treatment option for sepsis in children in the era of antibiotic resistance. Expert Rev. Anti. Infect. Ther. 12, 1237-1252.

Maifiah, M.H., Creek, D.J., Nation, R.L., Forrest, A., Tsuji, B.T., Velkov, T., and Li, J. 2017. Untargeted metabolomics analysis reveals key pathways responsible for the synergistic killing of colistin and doripenem combination against Acinetobacter baumannii. Sci. Rep. 7, 45527.

Malone, L. and Kwon, D.H. 2013. Carbapenem-associated multidrug-resistant Acinetobacter baumannii are sensitised by aztreonam in combination with polyamines. Int. J. Antimicrob. Agents $41,70-74$. 
Muller, G.L., Tuttobene, M., Altilio, M., Martinez Amezaga, M., Nguyen, M., Cribb, P., Cybulski, L.E., Ramírez, M.S., Altabe, S., and Mussi, M.A. 2017. Light modulates metabolic pathways and other novel physiological traits in the human pathogen Acinetobacter baumannii. J. Bacteriol. 199, e00011-17.

Mussi, M.A., Relling, V.M., Limansky, A.S., and Viale, A.M. 2007. $\mathrm{CarO}$, an Acinetobacter baumannii outer membrane protein involved in carbapenem resistance, is essential for L-ornithine uptake. FEBS Lett. 581, 5573-5578.

Naas, T., Cuzon, G., Bogaerts, P., Glupczynski, Y., and Nordmann, P. 2011. Evaluation of a DNA microarray (Check-MDR CT102) for rapid detection of TEM, SHV, and CTX-M extended-spectrum $\beta$-lactamases and of KPC, OXA-48, VIM, IMP, and NDM-1 carbapenemases. J. Clin. Microbiol. 49, 1608-1613.

Nait Chabane, Y., Marti, S., Rihouey, C., Alexandre, S., Hardouin, J., Lesouhaitier, O., Vila, J., Kaplan, J.B., Jouenne, T., and Dé, E. 2014. Characterisation of pellicles formed by Acinetobacter baumannii at the air-liquid interface. PLoS One 9, e111660.

Nemec, A., Krizova, L., Maixnerova, M., Van Der Reijden, T.J., Deschaght, P., Passet, V., Vaneechoutte, M., Brisse, S., and Dijkshoorn, L. 2011. Genotypic and phenotypic characterization of the Acinetobacter calcoaceticus-Acinetobacter baumannii complex with the proposal of Acinetobacter pittii sp. nov. (formerly Acinetobacter genomic species 3 ) and Acinetobacter nosocomialis sp. nov. (formerly Acinetobacter genomic species 13TU). Res. Microbiol. 162, 393-404.

Nepka, M., Perivolioti, E., Kraniotaki, E., Politi, L., Tsakris, A., and Pournaras, S. 2016. In vitro bactericidal activity of trimethoprimsulfamethoxazole alone and in combination with colistin against carbapenem-resistant Acinetobacter baumannii clinical isolates. Antimicrob. Agents Chemother. 60, 6903-6906.

Nikaido, H. 2003. Molecular basis of bacterial outer membrane permeability revisited. Microbiol. Mol. Biol. Rev. 67, 593-656.

Nikaido, H. 2009. Multidrug resistance in bacteria. Annu. Rev. Biochem. 78, 119-146.

Obara, M. and Nakae, T. 1991. Mechanisms of resistance to betalactam antibiotics in Acinetobacter calcoaceticus. J. Antimicrob. Chemother. 28, 791-800.

Pachon-Ibanez, M.E., Docobo-Perez, F., Lopez-Rojas, R., DominguezHerrera, J., Jimenez-Mejias, M.E., Garcia-Curiel, A., Pichardo, C., Jiménez, L., and Pachón, J. 2010. Efficacy of rifampin and its combinations with imipenem, sulbactam, and colistin in experimental models of infection caused by imipenem-resistant Acinetobacter baumannii. Antimicrob. Agents Chemother. 54, 11651172.

Pagano, M., Martins, A.F., and Barth, A.L. 2016. Mobile genetic elements related to carbapenem resistance in Acinetobacter baumannii. Braz. J. Microbiol. 47, 785-792.

Pankuch, G.A., Seifert, H., and Appelbaum, P.C. 2010. Activity of doripenem with and without levofloxacin, amikacin, and colistin against Pseudomonas aeruginosa and Acinetobacter baumannii. Diagn. Microbiol. Infect. Dis. 67, 191-197.

Petrosillo, N., Giannella, M., Antonelli, M., Antonini, M., Barsic, B., Belancic, L., Inkaya, A.C., De Pascale, G., Grilli, E., Tumbarello, M., et al. 2014. Clinical experience of colistin-glycopeptide combination in critically ill patients infected with Gram-negative bacteria. Antimicrob. Agents Chemother. 58, 851-858.

Poirel, L., Bonnin, R.A., and Nordmann, P. 2011. Genetic basis of antibiotic resistance in pathogenic Acinetobacter species. IUBMB Life 63, 1061-1067.

Post, V., White, P.A., and Hall, R.M. 2010. Evolution of AbaR-type genomic resistance islands in multiply antibiotic-resistant Acinetobacter baumannii. J. Antimicrob. Chemother. 65, 1162-1170.

Rafii, F., Sutherland, J.B., and Cerniglia, C.E. 2008. Effects of treatment with antimicrobial agents on the human colonic microflora. Ther. Clin. Risk Manag. 4, 1343-1358.

Rajamohan, G., Srinivasan, V.B., and Gebreyes, W.A. 2010. Mole- cular and functional characterization of a novel efflux pump, AmvA, mediating antimicrobial and disinfectant resistance in Acinetobacter baumannii. J. Antimicrob. Chemother. 65, 19191925.

Ramírez, M.S., Traglia, G.M., Pérez, J.F., Müller, G.L., Martínez, M.F., Golic, A.E., and Mussi, M.A. 2015. White and blue light induce reduction in susceptibility to minocycline and tigecycline in Acinetobacter spp. and other bacteria of clinical importance. J. Med. Microbiol. 64, 525-537.

Ribera, A., Roca, I., Ruiz, J., Gibert, I., and Vila, J. 2003. Partial characterization of a transposon containing the tet(A) determinant in a clinical isolate of Acinetobacter baumannii. J. Antimicrob. Chemother. 52, 477-480.

Roca, I., Espinal, P., Vila-Farrés, X., and Vila, J. 2012. The Acinetobacter baumannii oxymoron: commensal hospital dweller turned pan-drug-resistant menace. Front. Microbiol. 3, 148.

Roca, I., Marti, S., Espinal, P., Martínez, P., Gibert, I., and Vila, J. 2009. CraA, a major facilitator superfamily efflux pump associated with chloramphenicol resistance in Acinetobacter baumannii. Antimicrob. Agents Chemother. 53, 4013-4014.

Rodríguez, C.H., Nastro, M., Vay, C., and Famiglietti, A. 2015. In vitro activity of minocycline alone or in combination in multidrug-resistant Acinetobacter baumannii isolates. J. Med. Microbiol. 64, 1196-1200.

Rodríguez-Martínez, J.M., Nordmann, P., Ronco, E., and Poirel, L. 2010. Extended-spectrum cephalosporinase in Acinetobacter baumannii. Antimicrob. Agents Chemother. 54, 3484-3488.

Rosato, A., Piarulli, M., Corbo, F., Muraglia, M., Carone, A., Vitali, M.E., and Vitali, C. 2010. In vitro synergistic antibacterial action of certain combinations of gentamicin and essential oils. Curr. Med. Chem. 17, 3289-3295.

Scandorieiro, S., De Camargo, L.C., Lancheros, C.A., YamadaOgatta, S.F., Nakamura, C.V., De Oliveira, A.G., Andrade, C.G., Duran, N., Nakazato, G., and Kobayashi, R.K. 2016. Synergistic and additive effect of oregano essential oil and biological silver nanoparticles against multidrug-resistant bacterial strains. Front. Microbiol. 7, 760.

Schwarz, S., Kehrenberg, C., Doublet, B., and Cloeckaert, A. 2004. Molecular basis of bacterial resistance to chloramphenicol and florfenicol. FEMS Microbiol. Rev. 28, 519-542.

Shen, G.H., Wang, J.L., Wen, F.S., Chang, K.M., Kuo, C.F., Lin, C.H., and Luo, H.R., Hung, C.H. 2012. Isolation and characterization of $\varphi \mathrm{km} 18 \mathrm{p}$, a novel lytic phage with therapeutic potential against extensively drug resistant Acinetobacter baumannii. PLoS One 7, e46537.

Shields, R.K., Clancy, C.J., Gillis, L.M., Kwak, E.J., Silveira, F.P., Massih, R.C., and Eschenauer, G.A., Potoski, B.A., Nguyen, M.H. 2012. Epidemiology, clinical characteristics and outcomes of extensively drug-resistant Acinetobacter baumannii infections among solid organ transplant recipients. PLoS One 7, e52349.

Shields, R.K., Kwak, E.J., Potoski, B.A., Doi, Y., Adams-Haduch, J.M., Silviera, F.P., Toyoda, Y., Pilewski, J.M., Crespo, M., Pasculle, A.W., et al. 2011. High mortality rates among solid organ transplant recipients infected with extensively drug-resistant Acinetobacter baumannii: using in vitro antibiotic combination testing to identify the combination of a carbapenem and colistin as an effective treatment regimen. Diagn. Microbiol. Infect. Dis. 70, 246-252.

Shin, B. and Park, W. 2015. Synergistic effect of oleanolic acid on aminoglycoside antibiotics against Acinetobacter baumannii. PLoS One 10, e0137751.

Srinivasan, V.B., Rajamohan, G., and Gebreyes, W.A. 2009. Role of AbeS, a novel efflux pump of the SMR family of transporters, in resistance to antimicrobial agents in Acinetobacter baumannii. Antimicrob. Agents Chemother. 53, 5312-5316.

Stogios, P.J., Kuhn, M.L., Evdokimova, E., Law, M., Courvalin, P., and Savchenko, A. 2017. Structural and biochemical character- 
ization of Acinetobacter spp. aminoglycoside acetyltransferases highlights functional and evolutionary variation among antibiotic resistance enzymes. ACS Infect. Dis. 3, 132-143.

Su, X.Z., Chen, J., Mizushima, T., Kuroda, T., and Tsuchiya, T. 2005. AbeM, an $\mathrm{H}^{+}$-coupled Acinetobacter baumannii multidrug efflux pump belonging to the MATE family of transporters. Antimicrob. Agents Chemother. 49, 4362-4364.

Sugawara, E. and Nikaido, H. 2012. OmpA is the principal nonspecific slow porin of Acinetobacter baumannii. J. Bacteriol. 194, 4089-4096.

Sun, Y., Wang, L., Li, J., Zhao, C., Zhao, J., Liu, M., Wang, S., Lu, C., Shang, G., Jia, Y., et al. 2014. Synergistic efficacy of meropenem and rifampicin in a murine model of sepsis caused by multidrug-resistant Acinetobacter baumannii. Eur. J. Pharmacol. 729, $116-122$.

Tangden, T., Karvanen, M., Friberg, L.E., Odenholt, I., and Cars, O. 2017. Assessment of early combination effects of colistin and meropenem against Pseudomonas aeruginosa and Acinetobacter baumannii in dynamic time-kill experiments. Infect. Dis. (Lond.) 49, 521-527.

Temocin, F., Erdinc, F.S., Tulek, N., Demirelli, M., Ertem, G., Kinikli, S., and Koksal, E. 2015. Synergistic effects of sulbactam in multidrug-resistant Acinetobacter baumannii. Braz. J. Microbiol. 46, 1119-1124.

Thummeepak, R., Kitti, T., Kunthalert, D., and Sitthisak, S. 2016. Enhanced Antibacterial Activity of Acinetobacter baumannii bacteriophage OABP-01 endolysin (LysABP-01) in combination with colistin. Front. Microbiol. 7, 1402.

Tian, G.B., Adams-Haduch, J.M., Taracila, M., Bonomo, R.A., Wang, H.N., and Doi, Y. 2011. Extended-spectrum AmpC cephalosporinase in Acinetobacter baumannii: ADC-56 confers resistance to cefepime. Antimicrob. Agents Chemother. 55, 4922-4925.

Timurkaynak, F., Can, F., Azap, O.K., Demirbilek, M., Arslan, H., and Karaman, S.O. 2006. In vitro activities of non-traditional antimicrobials alone or in combination against multidrug-resistant strains of Pseudomonas aeruginosa and Acinetobacter baumannii isolated from intensive care units. Int. J. Antimicrob. Agents 27, 224-228.

Tiwari, V., Roy, R., and Tiwari, M. 2015. Antimicrobial active herbal compounds against Acinetobacter baumannii and other pathogens. Front. Microbiol. 6, 618.

Tomaras, A.P., Dorsey, C.W., Edelmann, R.E., and Actis, L.A. 2003. Attachment to and biofilm formation on abiotic surfaces by Acinetobacter baumannii: involvement of a novel chaperone-usher pili assembly system. Microbiology 149, 3473-3484.

Tran, J.H. and Jacoby, G.A. 2002. Mechanism of plasmid-mediated quinolone resistance. Proc. Natl. Acad. Sci. USA 99, 5638-5642.

Tsai, T., Chien, H.F., Wang, T.H., Huang, C.T., Ker, Y.B., and Chen, C.T. 2011. Chitosan augments photodynamic inactivation of Gram-positive and Gram-negative bacteria. Antimicrob. Agents Chemother. 55, 1883-1890.

Urban, C., Mariano, N., and Rahal, J.J. 2010. In vitro double and triple bactericidal activities of doripenem, polymyxin $\mathrm{B}$, and rifampin against multidrug-resistant Acinetobacter baumannii, Pseudomonas aeruginosa, Klebsiella pneumoniae, and Escherichia coli. Antimicrob. Agents Chemother. 54, 2732-2734.

Urban, C., Mariano, N., Rahal, J.J., Tay, E., Ponio, C., Koprivnjak, T., and Weiss, J. 2001. Polymyxin B-resistant Acinetobacter baumannii clinical isolate susceptible to recombinant BPI and ce- cropin P1. Antimicrob. Agents Chemother. 45, 994-995.

Valcourt, C., Saulnier, P., Umerska, A., Zanelli, M.P., Montagu, A., Rossines, E., and Joly Guillou, M.L. 2016. Synergistic interactions between doxycycline and terpenic components of essential oils encapsulated within lipid nanocapsules against Gram negative bacteria. Int. J. Pharm. 498, 23-31.

Vila, J., Ruiz, J., Goñi, P., and Jimenez de Anta, T. 1997. Quinoloneresistance mutations in the topoisomerase IV parC gene of Acinetobacter baumannii. J. Antimicrob. Chemother. 39, 757-762.

Wan, G., Ruan, L., Yin, Y., Yang, T., Ge, M., and Cheng, X. 2016. Effects of silver nanoparticles in combination with antibiotics on the resistant bacteria Acinetobacter baumannii. Int. J. Nanomedicine 11, 3789-3800.

Weber, B.S., Kinsella, R.L., Harding, C.M., and Feldman, M.F. 2017. The secrets of Acinetobacter secretion. Trends Microbiol. 25, 532545.

Wei, W.J. and Yang, H.F. 2017. Synergy against extensively drugresistant Acinetobacter baumannii in vitro by two old antibiotics: colistin and chloramphenicol. Int. J. Antimicrob. Agents 49, 321-326.

Wong, D., Nielsen, T.B., Bonomo, R.A., Pantapalangkoor, P., Luna, B., and Spellberg, B. 2017. Clinical and pathophysiological overview of Acinetobacter infections: a century of challenges. Clin. Microbiol. Rev. 30, 409-447.

Wu, X., Chavez, J.D., Schweppe, D.K., Zheng, C., Weisbrod, C.R., Eng, J.K., Murali, A., Lee, S.A., Ramage, E., Gallagher, L.A., et al. 2016. In vivo protein interaction network analysis reveals porinlocalized antibiotic inactivation in Acinetobacter baumannii strain AB5075. Nat. Commun. 7, 13414.

Yang, Y.S., Lee, Y., Tseng, K.C., Huang, W.C., Chuang, M.F., Kuo, S.C., Lauderdale, T.L., and Chen, T.L. 2016. In vivo and in vitro efficacy of minocycline-based combination therapy for minocycline-resistant acinetobacter baumannii. Antimicrob. Agents Chemother. 60, 4047-4054.

Yilmaz, G.R., Guven, T., Guner, R, Kocak Tufan, Z., Izdes, S., Tasyaran, M.A., and Acikgoz, Z.C. 2015. Colistin alone or combined with sulbactam or carbapenem against $A$. baumannii in ventilatorassociated pneumonia. J. Infect. Dev. Ctries. 9, 476-485.

Yoon, E.J., Balloy, V., Fiette, L., Chignard, M., Courvalin, P., and Grillot-Courvalin, C. 2016. Contribution of the Ade resistancenodulation-cell division-type efflux pumps to fitness and pathogenesis of Acinetobacter baumannii. MBio 7, e00697-16.

Yoon, E.J., Courvalin, P., and Grillot-Courvalin, C. 2013. RND-type efflux pumps in multidrug-resistant clinical isolates of Acinetobacter baumannii: Major role for AdeABC overexpression and AdeRS mutations. Antimicrob. Agents Chemother. 57, 2989-2995.

Yu, Y.S., Zhou, H., Yang, Q., Chen, Y.G., and Li, L.J. 2007. Widespread occurrence of aminoglycoside resistance due to ArmA methylase in imipenem-resistant Acinetobacter baumannii isolates in China. J. Antimicrob. Chemother. 60, 454-455.

Yun, S.H., Choi, C.W., Kwon, S.O., Park, G.W., Cho, K., Kwon, K.H., Kim, J.Y., Yoo, J.S., Lee, J.C., Choi, J.S., et al. 2011. Quantitative proteomic analysis of cell wall and plasma membrane fractions from multidrug-resistant Acinetobacter baumannii. J. Proteome Res. 10, 459-469.

Zhang, Y., Chen, F., Sun, E., Ma, R., Qu, C., and Ma, L. 2013. In vitro antibacterial activity of combinations of fosfomycin, minocycline and polymyxin B on pan-drug-resistant Acinetobacter baumannii. Exp. Ther. Med. 5, 1737-1739. 